\title{
Solid-Phase Synthesis of Isoalloxazines using Merrifield Resin
}

\author{
${\text { Geetanjali, }{ }^{a} \text { Ram Singh }}^{*, a, b}$ and S.M.S Chauhan ${ }^{a}$ \\ ${ }^{a}$ Department of Chemistry, University of Delhi, Delhi-110 007, India \\ ${ }^{b}$ Centre for Environmental Management of Degraded Ecosystems, School of Environmental Studies, \\ University of Delhi, Delhi - 110 007, India
}

\begin{abstract}
A reação da resina de Merrifield ligada a compostos diamino com aloxan monohidrato, resultou em uma resina ligada a isoaloxazinas 10-substituídas, que foram caracterizadas por fluorescência em fase sólida e espectroscopia de IV. A resina foi segmentada por solução aquosa de HF/DMF a 40\%, obtendo-se isoaloxazinas 7-carboxi-10-substituídas, que foram caracterizadas por UV-Vis., IV, ${ }^{1} \mathrm{H}$ RMN, fluorescência e análise elementar.
\end{abstract}

The reaction of Merrifield resin bound diamino compounds with alloxan monohydrate gave $7-$ resin bound 10-substituted isoalloxazines that were characterized by solid phase fluorescence and IR spectroscopy. The resin was cleaved by $40 \%$ aqueous HF/DMF to give 7-carboxy-10-substituted isoalloxazines that were characterized by UV-vis., IR, ${ }^{1} \mathrm{H}$ NMR, fluorescence and elemental analysis.

Keywords: isoalloxazines (or flavins), merrifield resin, polymer-supported synthesis, cyclocondensation, alloxan monohydrate, solid-state fluorescence

\section{Introduction}

Polymer-supported synthesis is an important tool in the synthesis of biologically active compounds including peptides, oligonucleotides and oligosaccharides. ${ }^{1,2}$ Further, the polymer-supported synthesis has also been applied in a variety of organic reactions including the synthesis of natural products, heterocycles and in medicinal chemistry. ${ }^{3-10}$

Synthesis of 10-substituted isoalloxazines by the acidic cyclocondensation of 2-substituted aminoanilines with alloxan monohydrate in aqueous and organic solvents has been reported by us. ${ }^{11-15}$ Now, we report an improved synthesis of selected novel 10-substituted isoalloxazine-7carboxylic acids via polymer-support to examine the feasibility of polymer-support synthesis in the synthesis of biologically active isoalloxazines. The isoalloxazines being a cofactor of flavoproteins, are involved in the catalysis of a wide variety of biological redox reactions, mediate electron transfer processes and in the regulation of neurotransmitters and detoxification of xenobiotics. ${ }^{15-19}$ The isoalloxazines are also found to possess anti-malarial activity and are potent inhibitors of both human and plasmodium glutathione reductase..$^{20,21}$

* e-mail: singh_dr_ram@yahoo.com

\section{Results and Discussion}

The reaction of chloromethylated polystyrene resin (1) with sodium salt of the 4-chloro-3-nitrobenzoic acid (2) gives the resin bound 4-chloro-3-nitrobenzoate (3). The reaction of substituted amines/anilines $\mathbf{4 a - 1}$ with $\mathbf{3}$ was carried out in DMF to get 4-(N-substituted amino)3-nitrobenzoate (5a-1). ${ }^{22}$ The reduction of $\mathbf{5 a - 1}$ with $\mathrm{SnCl}_{2} \cdot \mathrm{H}_{2} \mathrm{O}$ gave resin bound diamines $\mathbf{6 a - 1} .^{22}$ The each nucleophilic substitution products and the diamino products were cleaved with hydrofluoric acid (HF) in DMF and show satisfactory elemental analysis. The cyclocondensation of 6a-1 with alloxan monohydrate (7) in the presence of boric acid/acetic acid and DMF gave the resin bound isoalloxazines 8a-l (Scheme 1). The appearance of strong peaks in the region 16401660 and $1710-1730 \mathrm{~cm}^{-1}$ for the presence of carbonyl groups $(\mathrm{C}=\mathrm{O})$ at positions 2 and 4 respectively, and in the region $3460-3420 \mathrm{~cm}^{-1}$ for $-\mathrm{NH}$ group at position 3 in IR spectra is characteristic of the isoalloxazine ring. ${ }^{23}$ For example, the IR spectra of $\mathbf{8 a}$ shows peaks at 1729 , 1713 and $1661 \mathrm{~cm}^{-1}$ for three $\mathrm{C}=\mathrm{O}$ groups and at 3432 $\mathrm{cm}^{-1}$ for $-\mathrm{NH}$ group (Table 1) of the resin linked isoalloxazine. Further, the solid phase fluorescence ${ }^{24-27}$ of 8a-l shows emission spectra in the region 510-563 


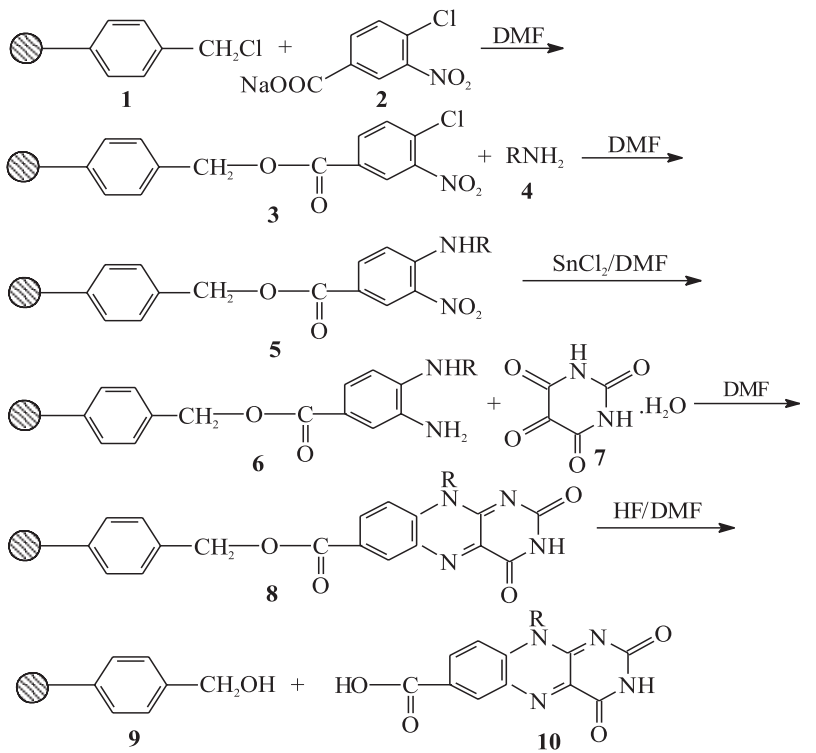

a. $\mathrm{CH}_{2} \mathrm{CH}_{2} \mathrm{CH}_{3}$; b. $\mathrm{CH}_{2}\left(\mathrm{CH}_{2}\right)_{2} \mathrm{CH}_{3}$; c. $\mathrm{CH}_{2}\left(\mathrm{CH}_{2}\right)_{8} \mathrm{CH}_{3}$;

d. $\mathrm{CH}_{2}\left(\mathrm{CH}_{2}\right)_{10} \mathrm{CH}_{3}$; e. $\mathrm{C}_{6} \mathrm{H}_{11}$; f. $\mathrm{CH}_{2} \mathrm{CH}=\mathrm{CH}_{2}$; g. $\mathrm{CH}_{2} \mathrm{C}_{6} \mathrm{H}_{5}$;

h. $\mathrm{C}_{6} \mathrm{H}_{5} ;$ i. 4' $^{\prime}-\mathrm{ClC}_{6} \mathrm{H}_{4} ;$ j. 4'- $-\mathrm{BrC}_{6} \mathrm{H}_{4}$; k. 4'- $-\mathrm{OCH}_{3} \mathrm{C}_{6} \mathrm{H}_{4}$ and l. 2', $6^{\prime}-\mathrm{CH}_{3} \mathrm{C}_{6} \mathrm{H}_{3}$

Scheme 1.

and $659-662 \mathrm{~nm}$ when excited at $420 \mathrm{~nm}$, which are very similar to their solution phase fluorescence spectra taken after cleavage at same excitation wavelength (Table 1, Experimental section). The reaction of 8a-1 with $40 \%$ aqueous HF/DMF gave 10 -substituted isoalloxazine-7-carboxylic acids (10a-1) in $74-81 \%$ yields. The structure of $\mathbf{1 0 a}-\mathbf{l}$ was confirmed by different spectroscopic data, including IR, UV-Visible, ${ }^{1} \mathrm{H}$ NMR, fluorescence and analytical data (experimental section).

In summary, the present polymer-supported synthesis is an attractive, clean, easy and efficient method in comparison to their solution phase synthesis where each synthetic step requires tedious purification process including column chromatography, for the synthesis of 7-carboxy-10-substituted isoalloxazines in good yields.

\section{Experimental}

All melting points are uncorrected and were recorded on Thomas Hoover Unimelt Capillary melting point Apparatus. IR spectra were recorded on Shimadzu TR-435 spectrophotometer $\left(v_{\max }\right.$ in $\left.\mathrm{cm}^{-1}\right)$. The absorption spectra were recorded on Shimadzu UV-260 spectrophotometer and absorption maxima were expressed in nm. ${ }^{1} \mathrm{H}$ NMR was recorded on Bruker Avance 300 spectrometer using TMS as an internal reference (chemical shift in ppm). Fluorescence spectra were recorded at the excitation value $420 \mathrm{~nm}$ on a Jobin Youn JY-3CS spectrofluorimeter and emission maxima were expressed in $\mathrm{nm}$. Elemental analysis was carried out in a Heraeus $\mathrm{CHN}$ analyzer.

Merrifield resin, Alloxan monohydrate and 4-chlorobenzoic acid were obtained from Across, Germany and used without further purifications.

Coupling of merrifield resin (1) with sodium salt of 4 chloro-3-nitrobenzoic acid (2)

1 (15 g) (chloromethylated styrene-divinylbenzene copolymer, $2 \%$ cross-linked, 2.50 mequiv of $\mathrm{Cl} / \mathrm{g}), 2$ (11.175 $\mathrm{g}, 50 \mathrm{mmol})$ and $\mathrm{DMF}(100 \mathrm{~mL})$ was taken and stirred for $24 \mathrm{~h}$. The suspension was filtered, washed subsequently with $\mathrm{H}_{2} \mathrm{O}, \mathrm{DMF}, \mathrm{CH}_{2} \mathrm{Cl}_{2}$ and $\mathrm{MeOH}(2 \times 100 \mathrm{~mL}$ each $)$ and dried in vacuum to get the resin $3(20.372 \mathrm{~g}) .2 .906 \mathrm{~g}(13 \mathrm{mmol})$ of the sodium salt was recovered after the reaction, which shows that approximately $36 \mathrm{mmol}$ is the theoretical loading capacity of the resin $3.15 .372 \mathrm{~g}$ of the resin $\mathbf{3}$ has been taken and divided into 12 equal parts to perform further reactions. The yields of the final compounds were calculated based on the theoretical loading.

General method for the synthesis of merrifield resin bound $\mathrm{N}$-substituted-2-nitroanilines (5a-l). Substituted amines/ anilines (4a-l) $(3.00 \mathrm{mmol})$ and DMF $(75 \mathrm{~mL})$ were added

Table 1.

\begin{tabular}{cccc}
\hline Compound & Yield / (g) & $\begin{array}{c}\text { Fluorescence } \\
\lambda \text { emission }^{\mathrm{a}} / \mathrm{nm}(\text { counts })\end{array}$ & $\begin{array}{c}\mathrm{IR}(\mathrm{KBr}) / \mathrm{cm}^{-1} \\
\mathrm{NH} \text { and C=O }\end{array}$ \\
\hline $\mathbf{8 a}$ & 1.358 & $510.0(3.15), 660.0(0.63)$ & 3432 and $1729,1713,1661$ \\
$\mathbf{8 b}$ & 1.356 & $521.0(7.78), 660.1(1.62)$ & 3442 and $1722,1709,1663$ \\
$\mathbf{8 c}$ & 1.551 & $517.9(4.49), 660.4(0.91)$ & 3432 and $1720,1710,1670$ \\
$\mathbf{8 d}$ & 1.613 & $513.8(2.29), 659.9(0.80)$ & 3436 and $1734,1702,1650$ \\
$\mathbf{8 e}$ & 1.529 & $523.2(7.79), 653.0(0.62)$ & 3460 and $1729,1711,1680$ \\
$\mathbf{8 f}$ & 1.341 & $518.8(3.39), 660.0(0.67)$ & 3425 and $1733,1715,1670$ \\
$\mathbf{8 g}$ & 1.439 & $518.4(0.99), 661.0(0.19)$ & 3445 and $1729,1715,1660$ \\
$\mathbf{8 h}$ & 1.388 & $560.6(6.69), 660.0(2.28)$ & 3455 and $1726,1713,1672$ \\
$\mathbf{8 i}$ & 1.415 & $563.4(9.97), 660.9(2.77)$ & 3442 and $1725,1715,1660$ \\
$\mathbf{8 j}$ & 1.571 & $513.0(2.59), 652.8(0.32)$ & 3425 and $1728,1712,1670$ \\
$\mathbf{8 k}$ & 1.411 & $559.0(12.97), 662.9(3.42)$ & 3428 and $1727,1711,1670$ \\
$\mathbf{8}$ & 1.476 & $510.9(3.29), 660.7(0.69)$ & 3432 and $1728,1718,1664$ \\
\hline
\end{tabular}

${ }^{a}($ Exc: $420 \mathrm{~nm})$. 
to each part of the resin (3) (1.281 g) at room temperature and the suspension was stirred at room temperature for $12 \mathrm{~h}$. It was filtered and washed subsequently with DMF, $\mathrm{CH}_{2} \mathrm{Cl}_{2}$ and $\mathrm{MeOH}(2 \times 50 \mathrm{~mL}$ each $)$ and dried in vacuo to get resin bound compounds (5a-l).

General method for the synthesis of merrifield resin bound $N$-substituted-2-aminoanilines $(\mathbf{6} \boldsymbol{a}-\boldsymbol{l})$. To each part of the resin bound nitro compounds (5-0.01 g) were added $\mathrm{SnCl}_{2} \cdot \mathrm{H}_{2} \mathrm{O}(3.50 \mathrm{mmol})$ and $\mathrm{DMF}(75 \mathrm{~mL})$ at room temperature. The suspensions was stirred at room temperature for $12 \mathrm{~h}$, filtered and washed subsequently with DMF, $\mathrm{H}_{2} \mathrm{O}, \mathrm{CH}_{2} \mathrm{Cl}_{2}$ and $\mathrm{MeOH}(2 \times 50 \mathrm{~mL}$ each $)$ and dried in vacuo to get resin bound diamino compounds (6a-l).

General method for the synthesis of 7-Merrifield resin bound 10 -substituted isoalloxazines $(8 \boldsymbol{a}-\mathbf{l})$. To each part of the resin 6a-l, alloxan monohydrate (7) (3.00 mmol), boric acid (3.00 $\mathrm{mmol})$, glacial acetic acid $(1.0 \mathrm{~mL})$ and DMF $(75 \mathrm{~mL})$ were added. The suspension was stirred at room temperature for $12 \mathrm{~h}$, filtered and washed with $\mathrm{H}_{2} \mathrm{O}, \mathrm{CH}_{2} \mathrm{Cl}_{2}$ and $\mathrm{MeOH}$ $(2 \times 50 \mathrm{~mL}$ each $)$ and dried in vacuo to get the resin $(\mathbf{8 a}-\mathbf{l})$.

Cleavage of resin bound isoalloxazines. To each part of the resin 8a-l, aqueous HF (40\%) and DMF (1:1) $(75 \mathrm{~mL})$ were added at $0{ }^{\circ} \mathrm{C}$ and stirred for $1 \mathrm{~h}$ at room temperature. The suspension was filtered and washed with DMF $(2 \times 50$ $\mathrm{mL})$. The combined filtrate was concentrated under reduced pressure to get the desired products $(\mathbf{1 0 a}-\mathbf{l})$ which was recrystallized from $\mathrm{AcOH} / \mathrm{H}_{2} \mathrm{O}$.

10-propylisoalloxazine-7-carboxylic acid (10a). Yield: $0.520 \mathrm{~g}$ (77\%); mp.: $>300{ }^{\circ} \mathrm{C}$; UV-Vis (DMSO) $\lambda_{\max } / \mathrm{nm}$ $\left(\varepsilon_{\max } / 10^{-3} \mathrm{~L} \mathrm{~mol}^{-1} \mathrm{~cm}^{-1}\right): 429$ (0.192), 346 (0.077), 285 (0.733); IR (KBr) $v_{\text {max }} / \mathrm{cm}^{-1}: 3404,3027,2846,2362,1713$, 1706, 1659, 1624, 1586, 1551, 1512, 1429, 1256, 1181, 1098, 884, 839, 774; ${ }^{1} \mathrm{H}$ NMR (DMSO- $\left.d_{6}\right), \delta 1.26(3 \mathrm{H}, \mathrm{t}$, $\left.J 6.3 \mathrm{~Hz}, \mathrm{CH}_{3}\right), 1.82-1.89\left(2 \mathrm{H}, \mathrm{m}, \mathrm{CH}_{2}\right), 4.67(2 \mathrm{H}, \mathrm{t}$, $\left.\mathrm{N}^{10} \mathrm{CH}_{2}, J 6.5 \mathrm{~Hz}\right), 7.78(1 \mathrm{H}, \mathrm{d}, \mathrm{H}-9, J 8.7 \mathrm{~Hz}), 8.06(1 \mathrm{H}$, bs, H-3), 8.48 (1H, d, H-8, J 9.0 Hz), 8.86 (1H, d, H-6, $J$ $1.5 \mathrm{~Hz}), 11.67$ (1H, s, COOH); Fluorescence (DMSO) $\lambda$ emission (intensity): 512.7 (4.32), 662.0 (0.77) nm; Elemental Analysis: Found,\%: C 56.05; H 4.09; N 18.79. $\mathrm{C}_{14} \mathrm{H}_{12} \mathrm{~N}_{4} \mathrm{O}_{4}$. Calculated,\%: C 56.00; H 4.03; N 18.66.

10-butylisoalloxazine-7-carboxylic acid (10b). Yield: 0.543 g (77\%); mp.: $>300{ }^{\circ} \mathrm{C}$; UV-Vis (DMSO) $\lambda_{\max } / \mathrm{nm}$ $\left(\varepsilon_{\max } / 10^{-3} \mathrm{~L} \mathrm{~mol}^{-1} \mathrm{~cm}^{-1}\right): 431$ (0.014), 334 (0.005), 288 (0.289); IR (KBr) $v_{\max } / \mathrm{cm}^{-1}: 3474,3169,2951,1720,1680$, 1660, 1591, 1556, 1519, 1405, 1345, 1250, 1236, 1099, 842, 771; ${ }^{1} \mathrm{H}$ NMR (DMSO- $\left.d_{6}\right), \delta 1.00(3 \mathrm{H}, \mathrm{t}, J 6.7 \mathrm{~Hz}$,
$\left.\mathrm{CH}_{3}\right), 1.52-1.79\left(4 \mathrm{H}, \mathrm{m},\left(\mathrm{CH}_{2}\right)_{2}\right), 4.61\left(2 \mathrm{H}, \mathrm{t}, \mathrm{N}^{10} \mathrm{CH}_{2}, J\right.$ $6.6 \mathrm{~Hz}), 7.94$ (1H, d, H-9, J 9.0 Hz), 8.09 (1H, bs, H-3), 8.40 (1H, d, H-8, J 8.7 Hz), 8.68 (1H, s, H-6), 11.60 (1H, $\mathrm{s}, \mathrm{COOH})$; Fluorescence (DMSO) $\lambda$ emission (intensity): 524.0 (9.90), 662.0 (2.23) nm.; Elemental Analysis: Found,\%: C 57.55; $\mathrm{H} 4.59 ; \mathrm{N} 17.83 . \mathrm{C}_{15} \mathrm{H}_{14} \mathrm{~N}_{4} \mathrm{O}_{4}$. Calculated,\%: C 57.50; H 4.50; N 17.88.

10-Decylisoalloxazine-7-carboxylic acid (10c). Yield: 0.700 $\mathrm{g}(78 \%)$; mp.: $>300{ }^{\circ} \mathrm{C}$; UV-Vis (DMSO) $\lambda_{\max } / \mathrm{nm}\left(\varepsilon_{\max } / 10^{-3}\right.$ $\mathrm{L} \mathrm{mol}^{-1} \mathrm{~cm}^{-1}$ ): 429 (1.533), 337 (0.790), 289 (2.385); IR (KBr) $v_{\text {max }} / \mathrm{cm}^{-1}: 3404,3027,2846,2362,1713,1706,1659,1624$, 1586, 1551, 1512, 1429, 1256, 1181, 1098, 884, 839, 774; ${ }^{1} \mathrm{H}$ NMR (DMSO- $\left.d_{6}\right), \delta 0.87\left(3 \mathrm{H}, \mathrm{t}, J 6.1 \mathrm{~Hz}, \mathrm{CH}_{3}\right), 1.61-$ $2.01\left(16 \mathrm{H}, \mathrm{m},\left(\mathrm{CH}_{2}\right)_{8}\right), 4.69\left(2 \mathrm{H}, \mathrm{t}, \mathrm{N}^{10} \mathrm{CH}_{2}, J 6.3 \mathrm{~Hz}\right), 7.65$ (1H, d, H-9, J 9.1 Hz), 8.55 (1H, d, H-8, J 8.9 Hz), 8.98 (1H, s, H-6), 11.80 (1H, s, COOH); Fluorescence (DMSO) $\lambda$ emission (intensity): 519.0 (5.73), 662.7 (0.99) nm; Elemental Analysis: Found,\%: C 63.40; $\mathrm{H}$ 6.59; $\mathrm{N}$ 14.03. $\mathrm{C}_{21} \mathrm{H}_{26} \mathrm{~N}_{4} \mathrm{O}_{4}$. Calculated,\%: C 63.30; H 6.58; N 14.06.

10-Dodecylisoalloxazine-7-carboxylic acid (10d). Yield: $0.759 \mathrm{~g}(79 \%)$; mp.: $>300{ }^{\circ} \mathrm{C}$; UV-Vis (DMSO) $\lambda_{\max } / \mathrm{nm}$ $\left(\varepsilon_{\max } / 10^{-3} \mathrm{~L} \mathrm{~mol}^{-1} \mathrm{~cm}^{-1}\right): 430$ (1.283), 347 (0.587), 285 (4.900); IR $(\mathrm{KBr}) v_{\max } / \mathrm{cm}^{-1}: 3404,3027,2846,2362,1713,1706$, 1659, 1624, 1586, 1551, 1512, 1429, 1256, 1181, 1098, 884, 839, 774; ${ }^{1} \mathrm{H}$ NMR (DMSO- $\left.d_{6}\right): 0.88\left(3 \mathrm{H}, \mathrm{t}, J 6.8 \mathrm{~Hz}, \mathrm{CH}_{3}\right)$, 1.87-2.11 (20H, m, $\left.\left(\mathrm{CH}_{2}\right)_{10}\right), 4.73\left(2 \mathrm{H}, \mathrm{t}, \mathrm{N}^{10} \mathrm{CH}_{2} J 6.7 \mathrm{~Hz}\right)$, 7.69 (1H, d, H-9, J 9.1 Hz), 8.58 (1H, d, H-8, J 8.0 Hz), 8.99 (1H, s, H-6), 11.89 (1H, s, COOH); Fluorescence (DMSO) $\lambda$ emission (intensity): 515.9 (3.70), 661.9 (1.14) nm; Elemental Analysis: Found,\%: C 64.72; $\mathrm{H} 7.07 ; \mathrm{N} 13.18 . \mathrm{C}_{23} \mathrm{H}_{30} \mathrm{~N}_{4} \mathrm{O}_{4}$. Calculated,\%: C 64.77; H 7.09; N 13.14.

10-Cyclohexylisoalloxazine-7-carboxylic acid (10e). Yield: $0.614 \mathrm{~g}(80 \%)$; mp.: $>300{ }^{\circ} \mathrm{C}$; UV-Vis (DMSO) $\lambda_{\text {max }} / \mathrm{nm}\left(\varepsilon_{\max }\right.$ I $\left.10^{-3} \mathrm{~L} \mathrm{~mol}^{-1} \mathrm{~cm}^{-1}\right): 432$ (1.787), 338 (0.812), 286 (9.454); IR (KBr) $v_{\max } / \mathrm{cm}^{-1}: 3404,3027,2846,2362,1713,1706,1659$, 1624, 1586, 1551, 1512, 1429, 1256, 1181, 1098, 884, 839, $774 ;{ }^{1} \mathrm{H}$ NMR (DMSO- $\left.d_{6}\right), \delta$ 1.25-2.00 $(10 \mathrm{H}, \mathrm{m}$, cyclohexyl$\mathrm{H})$, 4.15-4.17 (1H, m, $\left.\mathrm{N}^{10} \mathrm{CH}\right) 7.79(1 \mathrm{H}, \mathrm{d}, \mathrm{H}-9, J 8.9 \mathrm{~Hz})$, 8.21 (1H, bs, H-3), 8.56 (1H, d, H-8, J 8.9 Hz), 8.98 (1H, s, H-6), 11.86 (1H, s, COOH); Fluorescence (DMSO) $\lambda$ emission (intensity): 527.8 (8.63), $657.9(0.65) \mathrm{nm}$; Elemental Analysis: Found,\%: C 59.91; $\mathrm{H} 4.70 ; \mathrm{N} 16.42 . \mathrm{C}_{17} \mathrm{H}_{16} \mathrm{~N}_{4} \mathrm{O}_{4}$. Calculated,\%: C 59.99; H 4.74; N 16.46.

10-Allylisoalloxazine-7-carboxylic acid (10f). Yield: 0.538 g (80\%); mp.: >300 ${ }^{\circ} \mathrm{C}$; UV-Vis (DMSO) $\lambda_{\text {max }} / \mathrm{nm}\left(\varepsilon_{\max } /\right.$ $\left.10^{-3} \mathrm{~L} \mathrm{~mol}^{-1} \mathrm{~cm}^{-1}\right): 432$ (0.187), 344 (0.067), 286 (0.632); IR $(\mathrm{KBr}) v_{\max } / \mathrm{cm}^{-1}: 3412,3029,2846,2362,1720,1706$, 
$1680,1628,1586,1551,1512,1429,1256,1181,1098$ 884, 839 and $774 ;{ }^{1} \mathrm{H}$ NMR (DMSO- $\left.d_{6}\right), \delta 4.26-4.29(2 \mathrm{H}$, $\left.\mathrm{m}, \mathrm{N}^{10} \mathrm{CH}_{2}\right), 5.12-5.27\left(3 \mathrm{H}, \mathrm{m}, \mathrm{CH}=\mathrm{CH}_{2}\right), 7.78(1 \mathrm{H}, \mathrm{d}, \mathrm{H}-$ 9, J $8.7 \mathrm{~Hz}), 8.48(1 \mathrm{H}, \mathrm{d}, \mathrm{H}-8, J 9.0 \mathrm{~Hz}), 8.86(1 \mathrm{H}, \mathrm{s}, \mathrm{H}-$ 6), $11.67(1 \mathrm{H}, \mathrm{s}, \mathrm{COOH})$; Fluorescence (DMSO) $\lambda$ emission (intensity): 518.0 (4.38), $660.0(0.79) \mathrm{nm}$; Elemental Analysis: Found,\%: C 56.42; H 3.40; N 18.71. $\mathrm{C}_{14} \mathrm{H}_{10} \mathrm{~N}_{4} \mathrm{O}_{4}$. Calculated,\%: C 56.38; H 3.38; N 18.79.

10-Benzylisoalloxazine-7-carboxylic acid (10g). Yield: 0.620 $\mathrm{g}(79 \%)$; mp.: $>300{ }^{\circ} \mathrm{C}$; UV-Vis (DMSO) $\lambda_{\max } / \mathrm{nm}\left(\varepsilon_{\max } / 10^{-3}\right.$ $\left.\mathrm{L} \mathrm{mol}^{-1} \mathrm{~cm}^{-1}\right)$ : 422 (0.977), 342 (0.484), 268 (3.283); IR (KBr) $v_{\max } / \mathrm{cm}^{-1}: 3414,3182,3101,2924,2852,2570,2364,1729$, $1701,1623,1589,1457,1395,1363,1321,1273,1195,1148$, 872,$758 ;{ }^{1} \mathrm{H}$ NMR (DMSO- $d_{6}$ ), $\delta 5.94\left(2 \mathrm{H}, \mathrm{s}, \mathrm{N}^{10} \mathrm{CH}_{2}\right), 7.26-$ $7.54(5 \mathrm{H}, \mathrm{m}, \mathrm{Ar}-\mathrm{H}), 8.00(1 \mathrm{H}, \mathrm{d}, \mathrm{H}-9, J 8.9 \mathrm{~Hz}), 8.25(1 \mathrm{H}$, bs, H-3), 8.38 (1H, d, H-8, J 8.4 Hz), $8.84(1 \mathrm{H}, \mathrm{s}, \mathrm{H}-6)$, $12.01(1 \mathrm{H}, \mathrm{s}, \mathrm{COOH})$; Flourescence (DMSO) $\lambda$ emission (intensity): $521.4(1.78), 664.3(0.35) \mathrm{nm}$; Elemental Analysis: Found,\%: C 62.09; H 3.42; N 16.05. $\mathrm{C}_{18} \mathrm{H}_{12} \mathrm{~N}_{4} \mathrm{O}_{4}$. Calculated,\%: C 62.07; H 3.47; N 16.08 .

10-Phenylisoalloxazine-7-carboxylic acid (10h). Yield: 0.609 g (81\%); mp.: $>300{ }^{\circ} \mathrm{C}$; UV-Vis (DMSO) $\lambda_{\max } / \mathrm{nm}\left(\varepsilon_{\max } / 10^{-3}\right.$ $\left.\mathrm{L} \mathrm{mol}^{-1} \mathrm{~cm}^{-1}\right): 435$ (0.113), 334 (0.052), 285 (2.990); IR (KBr) $v_{\max } / \mathrm{cm}^{-1}: 3452,3220,3016,2926,1718,1654,1589,1545$, 1430, 1390, 1279, 1181, 907, 806, 767; ' $\mathrm{H}$ NMR (DMSO$\left.d_{6}\right), \delta 6.99(1 \mathrm{H}, \mathrm{d}, \mathrm{H}-9, J 8.2 \mathrm{~Hz}), 7.25-7.69(5 \mathrm{H}, \mathrm{m}, 10-$ phenyl H), $7.81(1 \mathrm{H}, \mathrm{d}, \mathrm{H}-8, J 8.1 \mathrm{~Hz}), 8.21(1 \mathrm{H}, \mathrm{d}, \mathrm{H}-6, J$ $1.9 \mathrm{~Hz}), 11.01(1 \mathrm{H}, \mathrm{s}, \mathrm{COOH})$; Fluorescence (DMSO) $\lambda$ emission (intensity): 567.0 (7.27), $662.0(2.68) \mathrm{nm}$; Elemental Analysis: Found,\%: C 61.03; H 2.99; N 16.77. $\mathrm{C}_{17} \mathrm{H}_{10} \mathrm{~N}_{4} \mathrm{O}_{4}$. Calculated,\%: C 61.08; H 3.01; N 16.76.

10-(4'-chlorophenyl)isoalloxazine-7-carboxylic acid (10i). Yield: $0.615 \mathrm{~g}(74 \%)$; mp.: $>300{ }^{\circ} \mathrm{C}$; UV-Vis (DMSO) $\lambda_{\text {max }} /$ $\mathrm{nm}\left(\varepsilon_{\max } / 10^{-3} \mathrm{~L} \mathrm{~mol}^{-1} \mathrm{~cm}^{-1}\right): 432$ (0.342), 338 (0.284), 286 (10.420); IR (KBr) $v_{\max } / \mathrm{cm}^{-1}: 3399,3173,3065,2936,1725$, 1665, 1585, 1544, 1399, 1289, 1192, 846 and; ${ }^{1} \mathrm{H}$ NMR (DMSO- $\left.d_{6}\right), \delta 7.62(1 \mathrm{H}, \mathrm{d}, \mathrm{H}-9, J$ 9.0 Hz), 7.74 (2H, d, H-2', H-6', J 9.0 Hz), 7.93 (2H, d, H-3', H-5', J 9.0 Hz), $8.31(1 \mathrm{H}$, dd, $\mathrm{H}-8, J 2.1$ and $9.0 \mathrm{~Hz}), 8.69(1 \mathrm{H}, \mathrm{bs}, \mathrm{H}-3), 8.92(1 \mathrm{H}, \mathrm{d}$, $\mathrm{H}-6, J 2.1 \mathrm{~Hz}), 11.90$ (1H, s, COOH); Fluorescence (DMSO) $\lambda$ emission (intensity): 567.0 (12.29), 663.9 (3.56) nm; Elemental Analysis: Found,\%: C 55.39; H 2.41; N 15.12. $\mathrm{C}_{17} \mathrm{H}_{9} \mathrm{~N}_{4} \mathrm{O}_{4} \mathrm{Cl}$. Calculated,\%: C 55.37; H 2.46; N 15.19.

10-(4'-Bromophenyl)isoalloxazine-7-carboxylic acid (10j). Yield: $0.689 \mathrm{~g}(74 \%)$; mp.: $>300{ }^{\circ} \mathrm{C}$; UV-Vis (DMSO) $\lambda_{\max } / \mathrm{nm}\left(\varepsilon_{\max } / 10^{-3} \mathrm{~L} \mathrm{~mol}^{-1} \mathrm{~cm}^{-1}\right): 431$ (0.857), 332 (0.289), 286 (1.246); IR (KBr) $v_{\text {max }} / \mathrm{cm}^{-1}: 3404,3027,2846$,
$2362,1713,1706,1659,1624,1586,1551,1512,1429$, $1256,1181,1098,884,839,774 ;{ }^{1} \mathrm{H}$ NMR (DMSO- $\left.d_{6}\right), \delta$ $7.64(1 \mathrm{H}, \mathrm{d}, \mathrm{H}-9, J 9.0 \mathrm{~Hz}), 7.76$ (2H, d, H-2', H-6', J 9.0 Hz), 7.92 (2H, d, H-3', H-5', J 9.0 Hz), 8.35 (1H, dd, H8, $J 2.1$ and $9.0 \mathrm{~Hz}), 8.72(1 \mathrm{H}, \mathrm{bs}, \mathrm{H}-3), 8.95(1 \mathrm{H}, \mathrm{d}, 6-\mathrm{H}$, $J 2.1 \mathrm{~Hz}), 11.89(1 \mathrm{H}, \mathrm{s}, \mathrm{COOH})$; Fluorescence (DMSO) $\lambda$ emission (intensity): 516.7 (3.11), $658.7(0.41) \mathrm{nm}$; Elemental Analysis: Found,\%: C 49.45; H 2.16; N 13.55. $\mathrm{C}_{17} \mathrm{H}_{9} \mathrm{~N}_{4} \mathrm{O}_{4} \mathrm{Br}$. Calculated,\%: C 49.42; H 2.19; N 13.56.

10-(4'-Methoxyphenyl)isoalloxazine-7-carboxylic acid (10k). Yield: $0.628 \mathrm{~g}(77 \%)$; mp.: $>300{ }^{\circ} \mathrm{C}$; UV-Vis (DMSO) $\lambda_{\max } / \mathrm{nm}\left(\varepsilon_{\max } / 10^{-3} \mathrm{~L} \mathrm{~mol}^{-1} \mathrm{~cm}^{-1}\right): 434$ (0.419), 340 (0.316), 282 (7.499); IR (KBr) $v_{\max } / \mathrm{cm}^{-1}: 3424,2925,1728$, 1662, 1640, 1607, 1490, 1459, 1299, 1219, 1176, 1040, $1017,833,757 ;{ }^{1} \mathrm{H}$ NMR (DMSO- $\left.d_{6}\right), \delta 4.06(3 \mathrm{H}, \mathrm{s}$, $\left.\mathrm{OCH}_{3}\right), 7.60(1 \mathrm{H}, \mathrm{d}, \mathrm{H}-9, J 9.6 \mathrm{~Hz}), 8.11(2 \mathrm{H}, \mathrm{d}, \mathrm{H}-3$ ', H5', J 9.3 Hz), 8.21 (2H, d, H-2', H-6', J 9.0 Hz), 8.29 $(1 \mathrm{H}, \mathrm{d}, \mathrm{H}-8, J 9.0 \mathrm{~Hz}), 8.80(1 \mathrm{H}, \mathrm{s}, \mathrm{H}-6), 11.92(1 \mathrm{H}, \mathrm{s}$, $\mathrm{COOH})$; Fluorescence (DMSO) $\lambda$ emission (intensity): 562.0 (15.97), 664.6 (4.02) nm; Elemental Analysis: Found,\%: C 59.37; $\mathrm{H} 3.35 ; \mathrm{N}$ 15.41. $\mathrm{C}_{18} \mathrm{H}_{12} \mathrm{~N}_{4} \mathrm{O}_{5}$. Calculated,\%: C 59.34; H 3.32; N 15.38.

10-(2, 6'-Dimethylphenyl)isoalloxazine-7-carboxylic acid (10l). Yield: $0.604 \mathrm{~g}(74 \%)$; mp.: $>300{ }^{\circ} \mathrm{C}$; UV-Vis (DMSO) $\lambda_{\text {max }} / \mathrm{nm}\left(\varepsilon_{\max } / 10^{-3} \mathrm{~L} \mathrm{~mol}^{-1} \mathrm{~cm}^{-1}\right): 431$ (0.038), 338 (0.023), 266 (0.130); IR (KBr) $v_{\max } / \mathrm{cm}^{-1}: 3422,3182,2926$, $1724,1664,1613,1588,1548,1460,1392,1272,1210$, 1187, 1025, 876, 796 and $745 ;{ }^{1} \mathrm{H}$ NMR (DMSO- $d_{6}$ ), $\delta$ $1.86\left(6 \mathrm{H}, \mathrm{s}, 2 \times \mathrm{CH}_{3}\right), 6.98(1 \mathrm{H}, \mathrm{d}, \mathrm{H}-9, J 8.0 \mathrm{~Hz}), 7.23-$ 7.29 (2H, m, 3'-H, 5'-H), 7.36-7.77 (2H, m, H-8, H-4'), $8.31(1 \mathrm{H}, \mathrm{d}, \mathrm{H}-6, J 2.1 \mathrm{~Hz}), 11.88(1 \mathrm{H}, \mathrm{s}, \mathrm{COOH})$; Flourescence (DMSO) $\lambda$ emission (intensity): 513.6 (4.68), 662.0 (0.96) nm; Elemental Analysis: Found,\%: C 62.95; $\mathrm{H}$ 3.85; $\mathrm{N}$ 15.47. $\mathrm{C}_{19} \mathrm{H}_{14} \mathrm{~N}_{4} \mathrm{O}_{4}$ Calculated,\%: $\mathrm{C}$ 62.98; $\mathrm{H}$ $3.89 ; \mathrm{N} 15.46$.

\section{Acknowledgments}

G. is thankful to CSIR, New Delhi, India for financial supports.

\section{References}

1. Bourne, G.T.; Meutermans, W.D.F.; Alewood, P.F.; McGeary, R.P.; Scanlon, M.; Watson, A.A.; J. Org. Chem. 1999, 64, 3095

2. Merrifield, R.B.; The Chemistry of Polypeptides; Katsoyannis, P.G., ed.; Plenum Press: New York, 1973, pp. 335-361.

3. Hermkens, P.H.H.; Ottenheijm, H.C.J.; Rees, D.C.; Tetrahedron 1997, 53, 5643. 
4. Gordeev, M.F.; Patel, D.V.; Gordon, E.M.; J. Org. Chem. 1996, 61,924 .

5. Sarshar, S.; Siev, D.; Mjalli, A.M.M.; Tetrahedron Lett. 1996, 37,835 .

6. Ruhland, B.; Bhandari, A.; Gordon, E.M.; Gallop, M.A.; J. Am. Chem. Soc. 1996, 118, 253.

7. Bunin, B.A.; Ellman, J.A.; J. Am. Chem. Soc. 1992, 114, 10997.

8. Morales, G.A.; Corbett, J.W.; DeGrado, W.F.; J. Org. Chem. 1998, 63, 1172.

9. Zajdel, P.; Subra, G.; Bojarski, A.J.; DuszyDska, B.; PawBowski, M.; Martinez, J.; Bioorg. Med. Chem. 2005, 13, 3029.

10. Makino, S.; Nakanishi, E.; Tsuji, T.; J. Braz. Chem. Soc. 2003 , $14,452$.

11. Chauhan, S.M.S.; Geetanjali; Singh, R; Indian J. Heterocy. Ch. 2000, 10, 157.

12. Geetanjali; Singh, R.; Chauhan, S.M.S.; Synth. Commun. 2003, 33,613 .

13. Chauhan, S.M.S.; Singh, R.; Geetanjali; Synth. Commun. 2003, $33,1179$.

14. Singh, R.; Geetanjali; Chauhan, S.M.S.; Bioorg. Chem. 2004, $32,140$.

15. Singh, R.; Geetanjali; Babu, C.R.; Chem. Biodivers. 2005, 2, 429.

16. Fitzpatrick, P.F.; Acc. Chem. Res. 2001, 34, 299.
17. Silverman, R.B.; The Organic Chemistry of Enzyme-Catalyzed Reactions, Acad. Press: New York, 2000, p.175.

18. Poulsen, L.L.; Zeigler, D.M.; Chem-Biol. Interact. 1995, 96, 57.

19. Berezovskii, V.M.; Tul'chinskaya, L.S.; Polyakova, N.A.; Russ. Chem. Rev. 1972, 41, 574.

20. Cowden, W.B.; Clark, I.A.; Hunt, N.H.; J. Med. Chem. 1988, $31,799$.

21. Cowden, W.B.; Butcher, G.A.; Clark, I.A.; Hunt, N.H.; Yoneda, F.; Am. J. Trop. Med. Hyg. 1987, 37, 495.

22. Lee, J.; Gauthier, D.; Rivero, R.A.; J. Org. Chem. 1999, 64, 3060 .

23. Hazekawa, I.; Nishina, Y.; Sato, K.; Shichiri, M.; Miura, R.; Shiga, K.; J. Biochemistry 1997, 121, 1147.

24. Pina-Luis, G.; Badia, R.; Diaz-Garcia, M. E.; Rivero, I. A. Jour.; Comb. Chem. 2004, 6, 391.

25. Castillo, M.; Rivero, I. A.; ARKIVOC 2003, xi, 193.

26. Hodge, P.; Synthesis and Separations using Functional Polymers; Sherrington, D.C., Hodge, P., eds.; John Wiley and Sons Ltd: New York, 1988: pp 43-122.

27. Walsh, D. P.; Pang, C.; Parikh, P.B.; Kim, Y.-S.; Chang, Y.-T. J.; Comb. Chem. 2002, 4, 204.
Received: January 7, 2005

Published on the web: February 6, 2006 